Ann. Biol. anim. Bioch. Biophys., I971, 11 (3), 363-37I.

\title{
STADES DU CYCLE DE L'ÉPITHÉLIUM SÉMINIFÈRE DU SINGE CRABIER, MACACA FASCICULARIS (= IRUS OU CYNOMOLGUS)
}

\author{
D.-C. DANG \\ Station centrale de Physiologie animale, \\ Centre national de Recherches zootechniques, I. N.R.A., \\ 78 - Jouy-en-Josas \\ Laboratoire d'Éthologie comparée, C. N.R. S., \\ 91 - Saint-Chéron \\ RÉSUMÉ
}

Quatre mâles pubères, âgés de 6 à 13 ans ont été hémicastrés, puis castrés à des époques différentes de l'année. L'étude a été faite sur coupes histologiques à $5 \mu$ ou $7 \mu$.

A la différence de l'Homme chez lequel l'épithélium séminifère montre un arrangement topographique particulier des associations des cellules germinales (CLERMONT, 1963), chez Macaca fascicularis la topographie de ces associations est semblable à celle connue chez tous les autres Mammifères. Nous avons divisé le cycle de l'épithélium séminifère en 8 stades selon OrTavant, (1958), décrit les différentes catégories de cellules germinales puis mis en évidence la constance de la fréquence relative des stades pour tous les testicules quels que soient l'âge des animaux ou les traitements subis (tabl. I).

Nous avons divisé le cycle en deux parties : la première partie commence dès la fin de la libération des spermatozoïdes dans la lumière des tubes séminifères et finit avec la dernière division homéotypique des spermatocytes II ; la seconde débute dès la fin de cette dernière division et se termine à la fin de l'élimination des spermatozoïdes dans la lumière du tube séminifère. Nous avons comparé ensuite ces deux parties avec celles des autres Primates déjà étudiés. Chez les Primates la répartition de ces deux parties est variable selon les espèces (tabl. 2).

\section{INTRODUCTION}

Un cycle de 1'épithélium séminifère montrant une succession régulière d'associations de cellules germinales a été retrouvé chez tous les Mammifères. Chez tous les Primates étudiés jusqu'ici à l'exception de l'Homme ; Microcebus murinus (PETTER, I969), Galagoides demidovii (VINCENT, I969), Macaca mulatta (CLERMONT, 
I957; CLERMONT et LEBLOND, I959; ARSENIEVA et al., I96I), Cercopithecus aethiops (ClERmont, I969), chaque section orthogonale de tube séminifère montre un seul type d'associations cellulaires. Chez l'Homme aucune section orthogonale ne montre un seul type d'associations mais de 3 à 6 aux limites malaisées à définir (CLERMONT, Ig63).

Devant les particularités bien frappantes de l'épithélium séminifère de l'Homme et étant donné le faible nombre d'espèces de Primates dont la spermatogenèse a été étudiée, nous avons entrepris l'étude de l'épithélium séminifère de Macaca fascicularis. Nous nous sommes attachés à examiner sa structure histologique, décrire les différentes catégories de cellules germinales, reconnaître les stades du cycle et déterminer leur fréquence.

\section{MATÉRIEI, ET TECHNIQUE}

Nous avons disposé de 4 animaux élevés au laboratoire depuis au moins 6 mois, recevant une alimentation variée ad libitum. Ces animaux âgés de 6 à I 3 ans étaient tous en bonne santé.

L'anesthésie a été obtenue par inhalation de protoxyde d'azote après immobilisation par une injection intramusculaire de solution aqueuse de chlorhydrate de I-(I-phényl-cyclohexyl) pipéridine (Sernyvet).

L'hémicastration et la castration ont été faites à des époques différentes de l'année.

Des cubes du parenchyme testiculaire de $6 \mathrm{~mm}$ d'épaisseur sont immédiatement immergés dans le fixateur. Nous avons utilisé 3 fixateurs : le Bouin Hollande, le Bouin sublimé et le Zenker formol. Pour tous ces fixateurs, la durée de fixation est de 3 jours à la température ambiante. Des coupes à $5 \mu$ ou $7 \mu$ d'épaisseur sont effectuées après inclusion dans la paraffine. Nous avons utilisé la coloration A. P. S. avec l'hématoxyline de Regaud et la coloration A. P. S. Feulgen.

\section{MÉTHODE D'ÉTUDE}

Nous avons choisi la classification des stades du cycle de l'épithélium séminifère utilisée par Roosen-Runge et Giesel. (1950) et Ortavant (1958). Ces stades sont ainsi définis :

Stade 1: de la fin de l'élimination des spermatozoïdes dans la lumière des tubes séminifères au début de l'élongation et l'augmentation de colorabilité du noyau des spermatides rondes.

Stade 2 : du début de l'élongation et de l'augmentation de colorabilité du noyau des spermatides rondes jusqu'au début de la formation en faisceaux de ces spermatides.

Stade $3:$ de la formation en faisceaux des spermatides au commencement de la première division hétérotypique des spermatocytes $\mathrm{I}$.

Stade 4: de la première division hétérotypique des spermatocytes I jusqu'à la dernière division homéotypique des spermatocytes II.

Stade 5: de la fin de la dernière division homéotypique des spermatocytes II jusqu'au moment où la chromatine du noyau des spermatides nouvellement formées devient poussiéreuse.

Stade 6 : depuis l'apparition des spermatides poussiéreuses jusqu'au moment où tous les faisceaux de spermatides de la génération précédente commencent leur migration centripète.

Stade $7:$ les spermatides de la génération précédente migrent et viennent border la lumière du tube séminifère.

Stade 8: les spermatozoïdes s'éliminent dans la lumière du tube.

Pour analyser les fréquences relatives des stades du cycle nous avons pris une coupe au hasard par morceau de testicule et sur cette coupe nous ne considérons que les tubes qui sont 
coupés orthogonalement sans tenir compte de la région du testicule. HochEREAU (I963) a pu montrer chez le Taureau que les fréquences relatives des stades ne sont pas affectées par la localisation de l'échantillon dans le testicule. Une photographie agrandie de la totalité de la coupe permet de noter les sections de tubes analysés.

\section{RÉSULTATS}

\section{I. - Topographie histologique de l'épithélium séminifère}

La topographie histologique de l'épithélium séminifère de Macaca fascicularis ressemble à celle de tous les Mammifères non humains dont la spermatogenèse a été étudiée. Chaque section orthogonale de tube séminifère ne présente qu'un ou deux stades bien définis du cycle. En coupe longitudinale les stades occupent un segment de tube et se succèdent dans le même ordre que dans le cycle de l'épithélium séminifère en constituant la vague spermatogénétique.

\section{2. - Détermination des stades}

Chez Macaca fascicularis la détermination selon la définition établie par ORTAVANT est facile. D'ailleurs la coloration A. P. S. révélant les différentes étapes de la spermiogenèse nous permet de prendre une décision rapide lorsqu'il s'agit de déterminer certains stades. De plus notre matériel nous a révélé d'autres précisions concernant les stades 2,5 et 7 .

Stade 2: les noyaux des spermatides, au début du stade, s'allongent et c'est à la fin du stade qu'ils prennent une coloration plus foncée sans se disposer en faisceaux.

Stade 5: les spermatides nouvellement formées grandissent vite, par suite il est difficile de différencier sans hésitation le stade 5 du stade 6 à partir de l'aspect des noyaux des jeunes spermatides rondes, il est nécessaire d'observer celui des spermatides allongées. En effet au stade 5 le noyau des spermatides allongées est plus foncé et plus brillant que celui des mêmes spermatides au stade 6. D'ailleurs cette décoloration progressive du noyau des spermatides allongées se poursuit jusqu'au stade 8 .

Stade 7: au début de la migration centripète des spermatides allongées certains noyaux des cellules de Sertoli accompagnent le faisceau avant de revenir près de la membrane basale. En plus on peut voir 1'apparition de fines sphères chromatophiles localisées dans le cytoplasme des spermatides allongées. Ces sphères chromatophiles se grouperaient pour former le corps résiduel laissé sur place par le spermatozoïde au moment de sa libération dans la lumière du tube séminifère au cours du stade 8 .

\section{3. - Les différents types de cellules germinales}

\section{Les spermatogonies.}

Spermatogonies $A$ foncées: leur noyau de diamètre $7,3 \mu$, généralement sphétique ou ovoide, renferme une chromatine en grains fins et une chromatine poussiéreuse très bien colorée. Il possède $I$ ou 2 nucléoles appliqués contre la membrane 
nucléaire et entourés d'une zone claire transparente. Le cytoplasme de ces cellules renferme de nombreux grains colorés en rose par l'A. P. S. Les spermatogonies A foncées se rencontrent à tous les stades du cycle, leur aspect et leur taille semblent ne pas se modifier au cours du cycle.

Spermatogonies $A$ claires: leur noyau plus grand (9-זo $\mu$ ) que celui des spermatogonies $\mathrm{A}$ foncées, s'aplatit légèrement sur la membrane basale. Les grains de chromatine, plus gros, se répartissent dans une chromatine poussiéreuse peu colorable. On compte de même I ou 2 nucléoles. Les spermatogonies A claires sont présentes aussi à tous les stades du cycle mais leur taille semble se modifier.

Spermatogonies $B$ : présentes du stade 3 au stade 8 , elles ont un noyau montrant des croûtelles de chromatine très colorable attachées à la membrane nucléaire et I ou 2 nucléoles bien visibles. Elles forment plusieurs générations successives car la taille de leur noyau diminue du stade 4 (12 $\mu$ ) au stade $8(9 \mu)$.

Les spermatocytes $I$.

Spermatocytes préleptotènes: leur noyau $(8 \mu)$ tout à fait rond avec des grosses croûtelles de chromatine qui ont tendance à se rassembler à un pôle ressemble beaucoup à celui des spermatogonies $B$ du stade 8 .

TABLEAU I

Comparaison des fréquences relatives des stades déterminées sur les sections orthogonales de tubes séminifères chez les 4 animaux

\begin{tabular}{|c|c|c|c|c|c|c|c|c|c|}
\hline \multirow[b]{2}{*}{ Animaux } & \multicolumn{9}{|c|}{ Stades } \\
\hline & 1 & 2 & 3 & 4 & 5 & 6 & 7 & 8 & $\begin{array}{l}\text { Nbre } \\
\text { total de } \\
\text { tubes }\end{array}$ \\
\hline Nbre de tubes ..... & 464 & 475 & 388 & 399 & 236 & 387 & 381 & 404 & \multirow{2}{*}{3134} \\
\hline P. $100 \ldots \ldots \ldots \ldots$ & 14,81 & 15,16 & 12,38 & 12,73 & 7,53 & 12,34 & 12,16 & 12,89 & \\
\hline Nbre de tubes ..... & 415 & 414 & 390 & 357 & 249 & 394 & 398 & 391 & \multirow{2}{*}{3008} \\
\hline P. $100 \ldots \ldots \ldots \ldots$ & 13,80 & 13,76 & 12,97 & 11,87 & 8,28 & 13,10 & 13,23 & 13,00 & \\
\hline Nbre de tubes ..... & 247 & 233 & 219 & 189 & 125 & 212 & 222 & 209 & \multirow{2}{*}{1656} \\
\hline P. $100 \ldots \ldots \ldots$ & 14,91 & 14,07 & 13,22 & 11,41 & 7,55 & 12,80 & 13,40 & 12,62 & \\
\hline Nbre de tubes ..... & 443 & 426 & 363 & 322 & 252 & 374 & 390 & 404 & \multirow{2}{*}{2984} \\
\hline P. $100 \ldots \ldots \ldots \ldots$ & 14,85 & 14,27 & 12,16 & 11,12 & 8,44 & 12,53 & 13,07 & 13,54 & \\
\hline$\chi^{2}$ calculé ......... & 1,97 & 3,63 & 3,57 & 5,12 & 6,52 & 3,87 & 4,31 & 2,00 & \multirow{2}{*}{10782} \\
\hline Signification ...... & NS & NS & NS & NS & NS & NS & NS & NS & \\
\hline \multirow{2}{*}{$\begin{array}{c}\text { Moyenne des } 4 \text { ani- } \\
\operatorname{maux} \ldots \ldots \ldots \ldots \ldots\end{array}$} & 14,54 & 14,31 & 12,68 & 11,78 & 7,95 & 12,69 & 12,96 & 13,01 & \\
\hline & $\pm 0,49$ & $\pm 0,54$ & $\pm 0,54$ & $\pm 0,54$ & $\pm 0,53$ & $\pm 0,54$ & $\pm 0,54$ & $\pm 0,54$ & \\
\hline
\end{tabular}


Spermatocytes leptotènes : leur noyau présente une masse de chromatine accolée à une partie de la membrane nucléaire d'où partent de fins filaments de chromatine spiralée.

Spermatocytes zygotènes: les fins chromosomes du stade leptotène se raccourcissent et s'associent deux à deux.

Spermatocytes pachytènes: au début du stade 4 ils sont dépourvus de nucléole visible et en possèdent un à partir du stade 5 .

Spermatocytes diplotènes: leurs chromosomes épais et courts présentent des entrecroisements perpendiculaires entre eux formant des chiasmata.

\section{Les spermatocytes $I I$.}

Ils sont présents uniquement au stade 4 , leur noyau ressemble à celui des spermatides rondes néoformées du stade 5 mais leur diamètre est nettement plus grand $(8 \mu)$.

Les spermatides rondes: au stade 5 leur noyau, de diamètre $6 \mu$, présente 4-5 croûtelles de chromatine bien colorées et à contours nets et des grains fins de chromatine qui semblent se disposer en réticule. Dans leur cytoplasme une zone plus ou moins sphérique, l'idiosome est très faiblement colorée par 1'A. P.S. et appliquée à un pôle du noyau. Au stade 6 le noyau de ces spermatides présentent 3-4 croûtelles de chromatine moins colorées, à contours flous et une chromatine poussiéreuse. L'idiosome possède 2-3 granules colorés par l'A. P. S. qui se groupent après pour former un seul granule appelé granule acrosomique. Au stade 7 le granule acrosomique s'aplatit légèrement et le capuchon acrosomique s'étale sur la membrane nucléaire en recouvrant le $I / 5$ du noyau et l'idiosome commence à se détacher du système acrosomique. Il en recouvre la moitié au stade $I$ et plus de la moitié à partir du stade 2 .

Les spermatides allongées: du stade 5 au stade 7 la colorabilité de leur noyau diminue progressivement tandis que leur cytoplasme s'écoule vers la partie postérieure. Au stade 8 ce sont de véritables spermatozoides qui s'éliminent en laissant sur place les gouttelettes cytoplasmiques ou corps résiduels.

\section{4. - La fréquence des stades du cycle de l'épithélium séminifère}

Les stades de I $\mathbf{5 4 0}$ sections orthogonales de tubes séminifères ont été déterminés pour chaque testicule, soit au total to 782 sections. Le tableau I indique pour les différents animaux la fréquence des stades. L'analyse statistique à l'aide du test de $\chi^{2}$ montre qu'il n'y a pas de différence significative entre les fréquences relatives de chaque stade des deux testicules d'un même animal, même 7 jours et 50 jours après l'hémicastration. De même elles ne varient pas d'un animal à un autre même si les animaux sont d'âge différent et castrés à des saisons différentes. 


\section{DISCUSSION}

\section{Topographie histologique}

La topographie de l'épithélium séminifère du macaque crabier, au point de vue histologique, est semblable à celle de tous les autres Primates déjà étudiés (1'Homme excepté). La coupe histologique de testicule du Babouin, Papio papio, publiée par STARK (I956) semble montrer qu'il existe chez cette espèce la structure histo-topographique classique des tubes séminifères des Mammifères; la section du tube correspondrait au stade 2 de notre classification. Nous remarquons la même structure chez Macaca nemestrina d'après la coupe de testicule publiée par CockerT et al. (1970), et chez Saimiri sciureus d'après la coupe publiée par Dumond (I968).

Si le cycle de l'épithélium séminifère existe bien chez l'Homme (CLERMONT, I963), la vague spermatogénétique serait, par contre, absente. La paroi des tubes séminifères de 1'Homme serait alors divisée en de très petits territoires ou unités qui évolueraient indépendamment les uns des autres. La structure de cette paroi est-elle unique chez 1'Homme ou existe-t-elle chez d'autres Primates non encore étudiés jusqu'ici? Rappelons qu'il existe quelques centaines d'espèces de Primates vivants et qu'on en a examiné que $g$ en ce qui concerne l'organisation topographique des cellules germinales de l'épithélium séminifère.

\section{Fréquences relatives des stades}

Pour les 7 testicules appartenant à 4 animaux nous avons trouvé les mêmes fréquences relatives des stades bien que ces testicules aient subi des traitements différents. Au sein d'une espèce de Primate les fréquences relatives des stades sont donc constantes, ce qui est en accord avec les résultats obtenus chez les Mammifères non Primates par divers auteurs : chez le Rat par LEBLOND et Ci,ermont (I952), Roosen-Runge (I950) et Hochereau (I963); chez le Bélier par OrTavant (I958); chez le Lapin par Swierstra et Foote (I963); chez le Taureau par AMmAN (I962) et HocherEAU (I963).

Il est difficile de comparer les fréquences des stades entre les espèces étudiées par différents auteurs car tous n'ont pas utilisé le même type de classification des stades ; en outre, comme le cycle de l'épithélium séminifère est un phénomène dynamique continu, l'appréciation du début d'un stade peut être différente suivant les auteurs. Or, dans les deux principales classifications, celle préconisée par LEBL,OND et CLERmon'T (I952) s'appuyant sur la spermiogenèse et la nôtre, il ressort que notre critère de détermination $\mathrm{du}$ stade 4 correspond exactement à celui qui caractérise

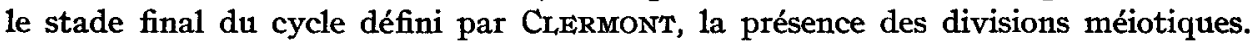
Il nous paraît donc intéressant de diviser le cycle en deux parties et de comparer chez les différentes espèces la somme des fréquences des stades se trouvant entre la fin de l'élimination des spermatozoïdes (début du stade $x$ ) et la fin des deuxièmes mitoses hétérotypiques (fin du stade 4 ) et celle des stades se trouvant entre la fin des deuxièmes mitoses hétérotypiques (début stade 5) et la fin de l'élimination des spermatozoïdes (fin du stade 8 ). Cette convention nous permet de comparer les 


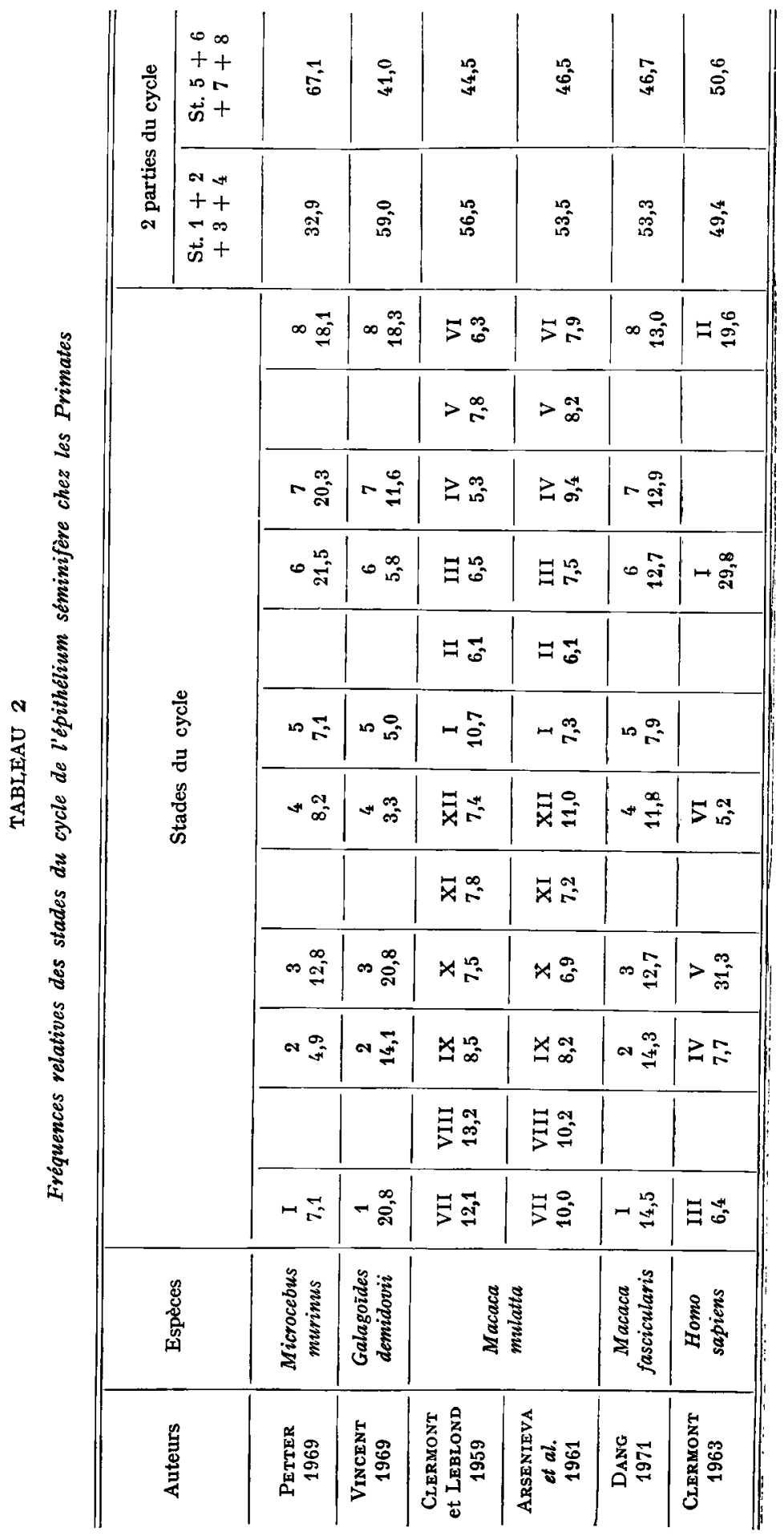


fréquences des stades de nos animaux avec celles des autres Primates. Le tableau 2 nous montre que chez Galagoides demidovii, Macaca et Homo sapiens les deux parties du cycle sont à peu près égales tandis que chez Microcebus murinus la première partie du cycle est deux fois plus courte que la seconde.

Cette différence dans la répartition des deux parties du cycle se retrouve aussichez des Mammifères non Primates. Chez le Rat et le Verrat la première partie est environ deux fois plus courte que la deuxième ; chez le Bélier et le Taureau la situation est exactement inverse ; tandis que chez le Lapin et 1'Éléphant ces deux parties du cycle sont sensiblement égales (comparaison faite par Thrbauli, I969).

Reçu pour publication en mars 1971.

\section{REMERCIEMEN'TS}

Nous remercions MM. Goustard, maître de recherches au C. N. R. S., PANIGEL et ThIBaUlT, professeurs à la Faculté des Sciences de Paris, pour l'aide qu'ils nous ont apportée dans l'accomplissement de ce travail.

\section{SUMMARY}

\section{THE STAGES OF THE SEMINIFEROUS EPITHELIUM CYCLE OF MACACA FASCICULARIS}

Four 6 to $\mathbf{I} 3$ years old mature male Macaca fascicularis (Irus or Cynomolgus) were hemicastrated, then castrated at various times of the year. Investigations were then carried out on 5 or $7 \mu$ serial sections.

The arrangement of germ cell associations in Macaca fascicularis was found similar to that of all other mammals and not in accordance with the peculiar arrangement observed in man (Clermont, r963).

The seminiferous epithelium cycle was divided into 8 stages according to ORTAVANT (I968). The various cell types were described and it was found that the relative occurence frequency of the stages was constant regardless of the age or treatment of the testes (Table I).

We divided the cycle into 2 parts. The first part includes stages I to 4 (from the achievement of the liberation of spermatozoa into the lumen of seminiferous tubules to the last meiotic division) ; the second part includes stages 5 to 8 .

A comparison of the relative duration of the two parts with other investigated primates showed it varies depending on the species (see table 2).

\section{RÉFÉRENCES BIBLIOGRAPHIQUES}

Ammann R. P., 1962. Reproductive capacity of dairy bulls. Amer. J. Anat., 110, 69-78.

Arsenieva N. A., et al., r96r. A radiation analysis of the duration of meiosis phases in the spermatogenesis of Macaca mulatta (russe). Dokl. Akad. Nauk. S.S.S. R., 141, I486-1489.

Crermont $Y$., 1957. The pattern of spermatogonial renewal in the seminiferous epithelium of the monkey. Anat. Rec., 127, 279.

Clermont Y., I963. The cycle of the seminiferous epithelium in man. Amer. J. Anat., 112, 35-5I.

Ciermont $Y ., 1969$. Two classes of spermatogonial stem cells in the monkey, Cercopithecus aethiops Amer. J, Anat., 126, 5 I-7I.

Clermont Y., Leblond C. P., r959. Differenciation and renewal of spermatogonia in the monkey, Macacus rhesus. Amer. J. Anat., 104, 237-272. 
Cocketт A. T. K., et al., 1970. The effects of immobilization on spermatogenesis in subhuman primates. Fertil. Steril., 21, 6ro-6r4.

Dumond F. V., I968. The squirrel monkey in a seminatural environment. In : The squirrel monkey éd. Rosenblum and Cooper.

Hochereau M. T., I963. Constance des fréquences relatives des stades du cycle de l'épithélium séminifère chez le Taureau et chez le Rat. Ann. Biol. anim. Bioch. Biophys., 3, 93-1oz.

LeBLoND C. P., CLERMONT Y., 1952. Definition of the stages of cycle of the seminiferous epithelium of the rat, Ann. N. Y. Acad. Sci., 55, 548-573.

Ortavant R., I958. Le cycle spermatogénétique chez le Bélier. Thèse Fac. Sci., Paris.

Petter A., r969. Cité in : Testicular physiology and biochemistry. Éd. A. D. Johnson, W. R. Gomes, N. L. Vandermark, Acad. Press.

Roosen-Runge E. C., Giesel L. O., 1950. Quantitative studies on spermatogenesis in the albino Rat. Amer. J. Anat., 87, 1-3o.

Stark O., I956. In : Primatologia, vol. I, éd. Hofer et al.

Swierstra E. E., Foote R. H., I963. Cytology and kinetics of spermatogenesis in the rabbit. $J$. Reprod. Fertil., 5, 309-322.

Thibault C., r969. La spermatogenèse chez les Mammifères. In : Traité de Zoologie. T. XVI, fascicule VI. Éd. P. P. Grassé, Masson et Cie, Paris.

Vincent F., ig69. Contribution à l'étude des prosimiens africains: le Galago de Demidoff. Thèse Fac. Sci., Paris. 
PLANCHE I

Stade du cycle de l'épithélium séminifère

FIG. I. - Stade I

L,es spermatozoīdes ont été éliminés dans la lumière du tube séminifère. Les spermatides possèdent un noyau rond.

FIG. 2. - Stade 2

Les noyaux des spermatides s'allongent.

Frg. 3. - Stade 3

Les spermatides allongées forment des faisceaux dont la pointe se dirige vers la membrane basale.

FIG. 4. - Stade 4

Apparition des divisions méiotiques des spermatocytes II et des spermatides néoformées. 

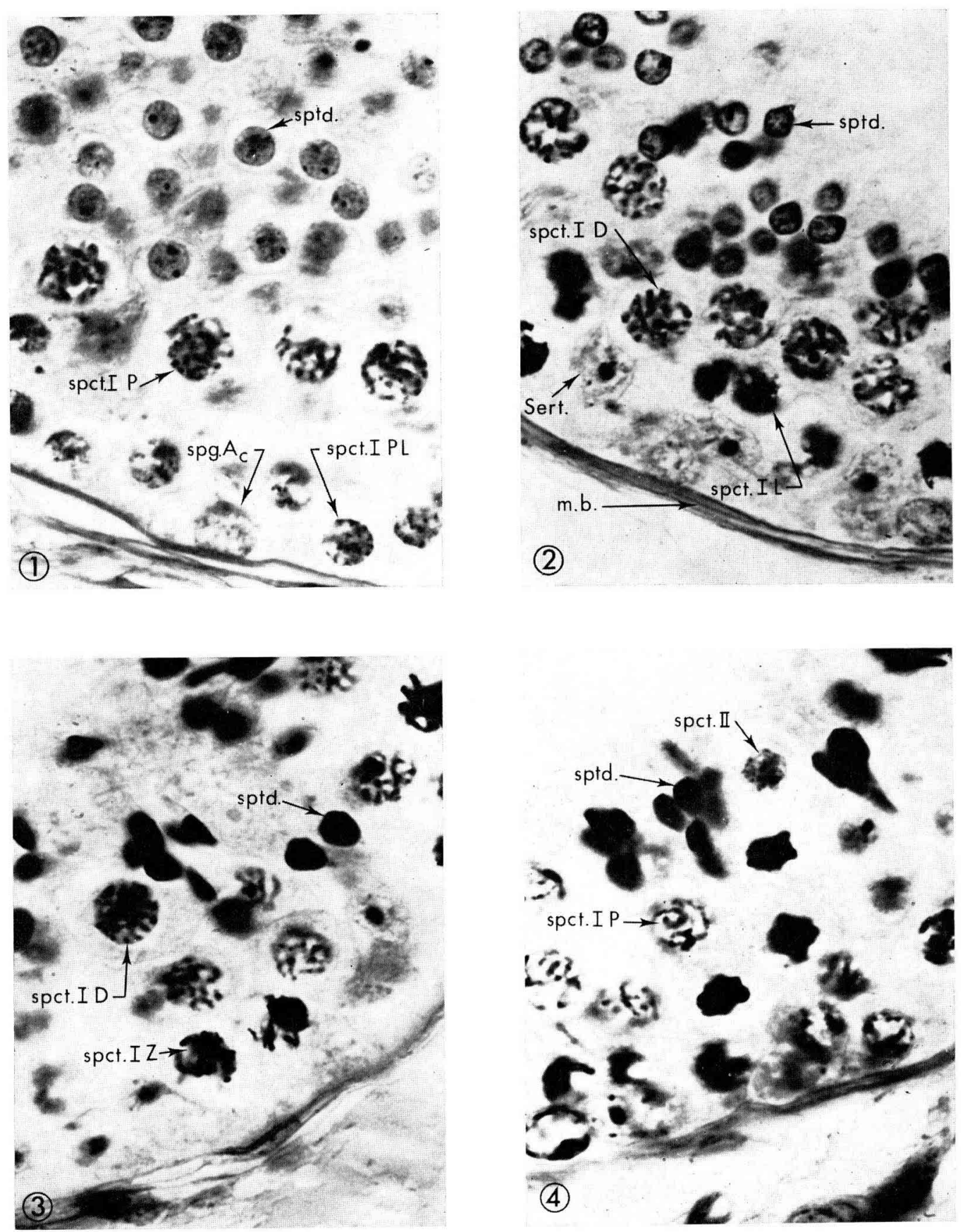
Fig. 5. - Stade 5. Le noyau des jeunes spermatides présente de nombreuses croutelles de chromatine. Les vieilles spermatides allongées ont leur noyau intensément coloré et brillant.

Fig. 6. - Stade 6. La chromatine des noyaux des jeunes spermatides devient poussiéreuse. Les vieilles spermatides restent en "copulation" avec les cellules de Sertoli.

Fig. 7. - Stade 7. Les faisceaux de vieilles spermatides effectuent un mouvement centripète. Leur cytoplasme commence à s'éliminer en de fines sphères.

FIG. 8. - Stade 8. Les spermatozoïdes s'éliminent dans la lumière du tube séminifère.

m. b. : membrane basale ; Sert. : cellule de Sertoli ; spg. Af : spermatogonie A foncée; spg. Ac : spermatogonie A claire; spg. Bx : spermatogonie B de génération inconnue ; spct. I PL : spermatocyte primaire préleptotène; spct. I L : spermatocyte primaire leptotène ; spct. I $Z$ : spermatocyte primaire zygotène ; spct. I D : spermatocyte primaire diplotène ; spct. II : spermatocyte secondaire ; sptd : spermatide ; spz. : spermatozoïde; gtt. cyt. : gouttelette cytoplasmique. 

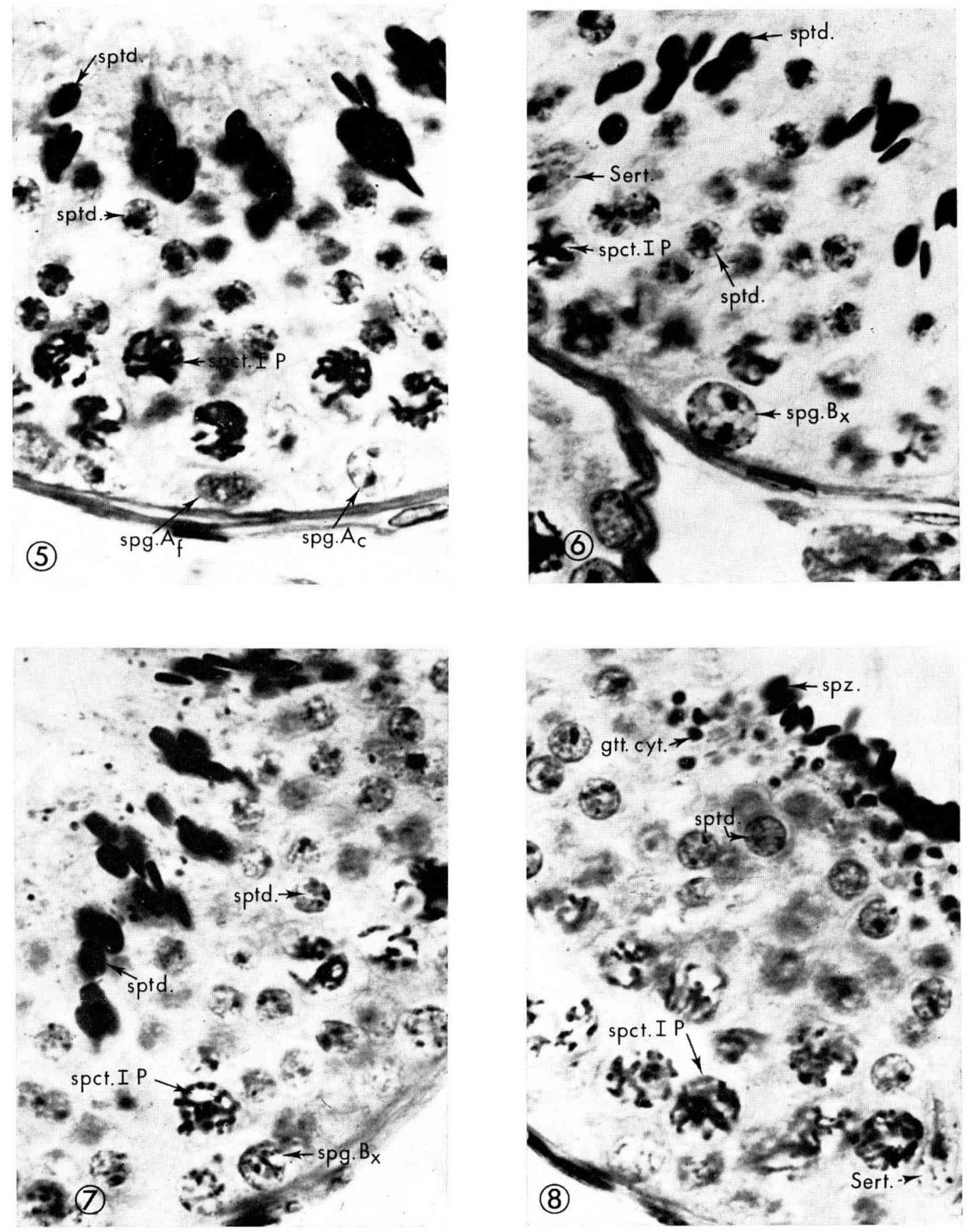

D.-C. DANG 